Collection SFN 9 (2008) 159-179

(C) EDP Sciences, Les Ulis

DOI: $10.1051 /$ sfn:2008012

\title{
Diffusion neutronique sous conditions extrêmes
}

\author{
J.-M. Mignot \\ Laboratoire Léon Brillouin, CEA-CNRS, CEA/Saclay, 91191 Gif-sur-Yvette, France
}

\begin{abstract}
Résumé. Depuis quelques années, on assiste dans tous les centres de neutrons à un regain d'intérêt pour les expériences réalisées sous "conditions extrêmes". En partant des techniques classiques de basses températures, forts champs et hautes pressions adaptées à la diffusion neutronique, ce cours passe en revue les développements en cours ou à venir, depuis les échantillons microscopiques étudiés dans les cellules à enclumes en diamant jusqu'aux puissantes installations devant produire des champs magnétiques bien supérieurs à la limite actuelle des 15 teslas.
\end{abstract}

\section{INTRODUCTION}

L'intérêt des scientifiques pour les conditions extrêmes n'est pas récent. On peut sans doute le faire remonter aux premiers travaux sur la liquéfaction des gaz, dont celle du dioxyde de soufre par Clouet et Monge en 1780 et celle de l'ammoniac par van Marum and vanTroostwijken en 1787. Ces succès marquent le début d'une quête de plus d'un siècle qui aboutira à la première production d'hélium liquide par Kamerlingh Onnes en 1908, bientôt suivie de la découverte de la supraconductivité dans le mercure. Par l'application de conditions extrêmes, l'expérimentateur peut envisager non seulement d'étendre la gamme de paramètres accessible pour la mesure de certaines grandeurs physiques et la vérification de théories existantes, mais aussi de faire apparaître des états entièrement nouveaux qui viendront enrichir notre connaissance des propriétés de la matière. Avec les progrès de l'instrumentation, ce type de travaux connaît un essor continu dans les laboratoires de recherche fondamentale, sur des sujets allant des fluides et solides quantiques ou des systèmes d'électrons fortement corrélés jusqu'aux sciences de la Terre et à la biologie. Il en résulte un accroissement du nombre de travaux mettant en jeu des valeurs inhabituelles des paramètres thermodynamiques température, champ magnétique ou pression. La diffusion des neutrons ne fait pas exception : technique essentielle pour les études structurales et dynamiques du réseau cristallin et du magnétisme, elle a su se mettre à l'heure des conditions extrêmes et se doter elle aussi de moyens adaptés pour contribuer aux avancées en cours dans ce domaine.

Les mesures sous conditions extrêmes ont longtemps été l'apanage de laboratoires spécialisés dans la cryogénie, les champs forts, les hautes pressions, etc., disposant d'un savoir-faire et de moyens techniques spécifiques pour construire et mettre en œuvre les dispositifs appropriés. Avec le temps, ces techniques ont fini par diffuser dans un grand nombre de laboratoires et, pour certaines, par donner lieu à des appareillages semi-industriels commercialisés par des entreprises de haute technologie. Les expériences de neutrons, réalisées dans des réacteurs ou des sources à spallation vont devoir faire face à des contraintes particulières, à la fois techniques — accés du faisceau à l'échantillon — et pratiques exploitation dans le cadre d'un service à des utilisateurs de Très Grands Équipements (TGE) ne disposant en général que d'un temps d'accés limité aux instruments. Ce handicap vis à vis des techniques "de laboratoire" a pu être en grande partie surmonté grâce aux avancées techniques réalisées ces derniéres années, notamment en matière de champs magnétiques et de hautes pressions. On verra qu'à l'inverse, les neutrons disposent de plusieurs atouts précieux par rapport aux techniques concurrentes. L'objectif de ce cours va être de donner un aperçu des moyens expérimentaux offerts aux utilisateurs des grands centres de neutrons pour réaliser des expériences sous conditions extrêmes, avec leurs limites actuelles et les progrés prévisibles. Cependant, plutôt que de m'en tenir à la simple description des techniques et des appareillages, je l'illustrerai d'exemples empruntés à des études récentes afin de 
mieux faire ressortir le potentiel que ceux-ci représentent pour différents domaines d'étude de la matière condensée. Le choix est bien sûr subjectif et il ne prétend nullement refléter l'importance relative des différents travaux.

Dans ce qui suit, le terme de conditions extrêmes sera pris dans le sens restrictif de : "basses températures et/ou forts champs magnétiques et/ou hautes pressions". On ne traitera donc, entre autres, ni du champ électrique ni des contraintes uniaxiales [21]. Les environnements d'échantillons pour les hautes températures sont évoqués dans l'article de Bayley [2], oú le lecteur trouvera aussi des informations complémentaires sur les sujets abordés dans ce cours.

\section{MATÉRIAUX}

En tant que moyen d'étude de la matière condensée, les neutrons se distinguent d'autres sondes comme les électrons ou les rayons X par un important pouvoir de pénétration dans toute une gamme de matériaux (Fig. 1). Cependant, dans le cas d'environnements d'échantillons complexes (écrans thermiques, bobinages d'électroaimants, parois de chambres de pression), les épaisseurs mises en jeu peuvent devenir significatives, et le concepteur devra porter une attention particulière à la géométrie des différents éléments et aux matériaux qui les constituent. Dans le choix de ceux-ci, il devra prendre en compte l'atténuation du faisceau qui les traverse, les signaux de diffusion parasite qu'ils peuvent générer, ainsi que les risque d'activation tant des constituants nominaux que d'éventuelles impuretés. Les alliages d'aluminium (2017 A, 5056 A, 5754 X) sont largement utilisés pour leur grande transparence aux neutrons (section efficace d'absorption $\sigma_{a}(\mathrm{Al})=0,231$ barn à $\lambda=1,798 \AA$ [35]). La section efficace incohérente de l'aluminium étant elle aussi très faible $\left(\sigma_{i}=0,256\right.$ barn $)$, seule la diffusion cohérente peut donner des effets gênants, notamment des "raies de poudre" parasites dans les diffractogrammes. Pour s'en prémunir, on pourra opter pour le vanadium, métal usinable offrant une section efficace cohérente voisine de zéro $\left(\sigma_{c}=0,018\right.$ barn), mais au prix d?une diffusion incohérente significative. Lorsqu'une plus grande résistance mécanique est requise, on choisit des alliages d'aluminium à hautes performances (7049 A autour de l'ambiante, 7075 à basse température), ou un alliage Ti-Zr à "matrice nulle" qui réalise une compensation entre les longueurs de diffusion cohérentes $b_{c}$ de signes opposés $\left(b_{c}^{\mathrm{Ti}}=-3,438 \mathrm{fm}, b_{c}^{\mathrm{Zr}}=7,16 \mathrm{fm}\right)$ de ses éléments constitutifs pour la concentration (en poids) $\mathrm{Ti}_{0,525} \mathrm{Zr}_{0,475}$.

En tout état de cause on s'efforcera, dés le stade de la conception, de réduire les épaisseurs de matière traversées par le faisceau. Nous en verrons quelques exemples dans la suite (bobines de type

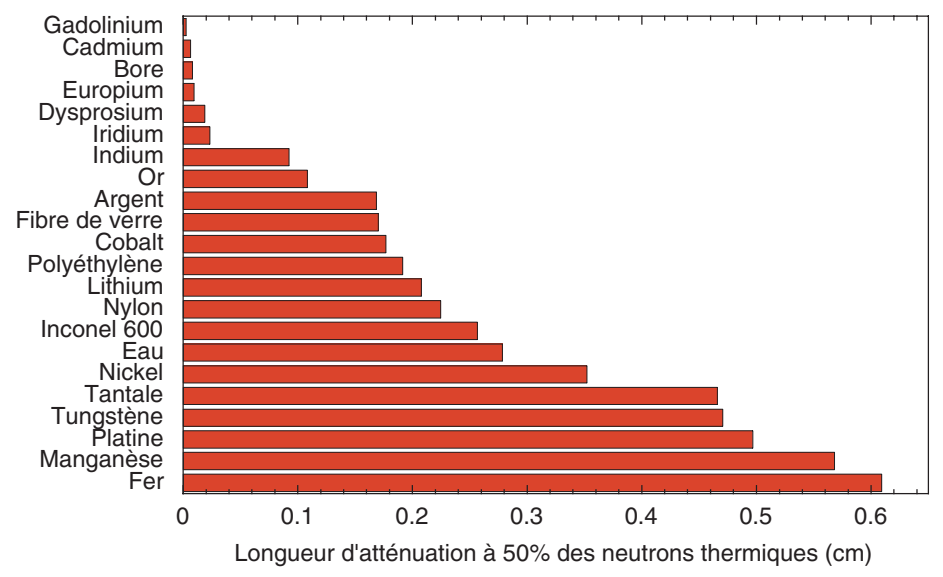

Figure 1. Longueurs caractéristiques d'atténuation à 50\% dans différents matériaux pour des neutrons d'énergie $25.3 \mathrm{meV}$ (en prenant comme référence la section efficace totale [35]). 
Helmholtz, chambres de pressions "panoramiques"). On veillera aussi à minimiser l'exposition aux neutrons d'éléments susceptibles de les rediffuser et/ou de s'activer, que ce soit en collimatant et en diaphragmant le faisceau incident ou en recouvrant leurs surfaces de matériaux absorbants (feuilles de cadmium, peinture au gadolinium). Il va sans dire, enfin, que dans le cas des systèmes cryogéniques contenant les fluides (azote, hélium, etc.), les réservoirs ne devront en aucun cas se trouver sur le trajet des neutrons.

\section{BASSES TEMPÉRATURES}

\subsection{Au-dessus de $1 \mathrm{~K}$ : cryostats à flux d'hélium et cryogénérateurs}

Pour les températures allant jusqu'à 1,5 K, l'appareillage le plus répandu dans les centres de neutrons est le cryostat à flux d'hélium connu sous le nom de "cryostat orange". Développé initialement à l'ILL à Grenoble [9], il est maintenant commercialisé par une société britannique (Fig. 2, à gauche). D'autres fabricants ont à leur catalogue des matériels similaires. L'échantillon, placé dans une enceinte contenant un gaz d'échange statique, est refroidi par un échangeur dans lequel circule de l'hélium prélevé sur le bain principal à travers une vanne à pointeau. Les fluides cryogéniques, hélium et azote, sont contenus dans des réservoirs situés au-dessus du trajet du faisceau, qui n'a donc à traverser que des écrans anti-radiations en alliage d'aluminium. La température de l'échangeur peut être stabilisée à volonté au moyen d'un régulateur classique à PID (proportionnel-intégral-dérivé). Les changements d'échantillon s'effectuent rapidement par simple extraction de la canne porte-échantillon ("sample stick").

Ce type de cryostat se voit de plus en plus concurrencé par une nouvelle génération de cryogénérateurs, ou "réfrigérateurs en circuit fermé" (également désignés par l'appellation commerciale de Displex). Fonctionnant par oscillation d'un volume d'hélium gazeux qui échange périodiquement de la chaleur avec un "régénérateur" $\left(\mathrm{Pb}, \mathrm{Er}_{3} \mathrm{~N}\right.$ ou $\left.\mathrm{NdAg}\right)$ suivant un cycle thermodynamique de Gifford-McMahon (GMM), ces appareillages existent aujourd'hui dans des versions à un étage, pouvant
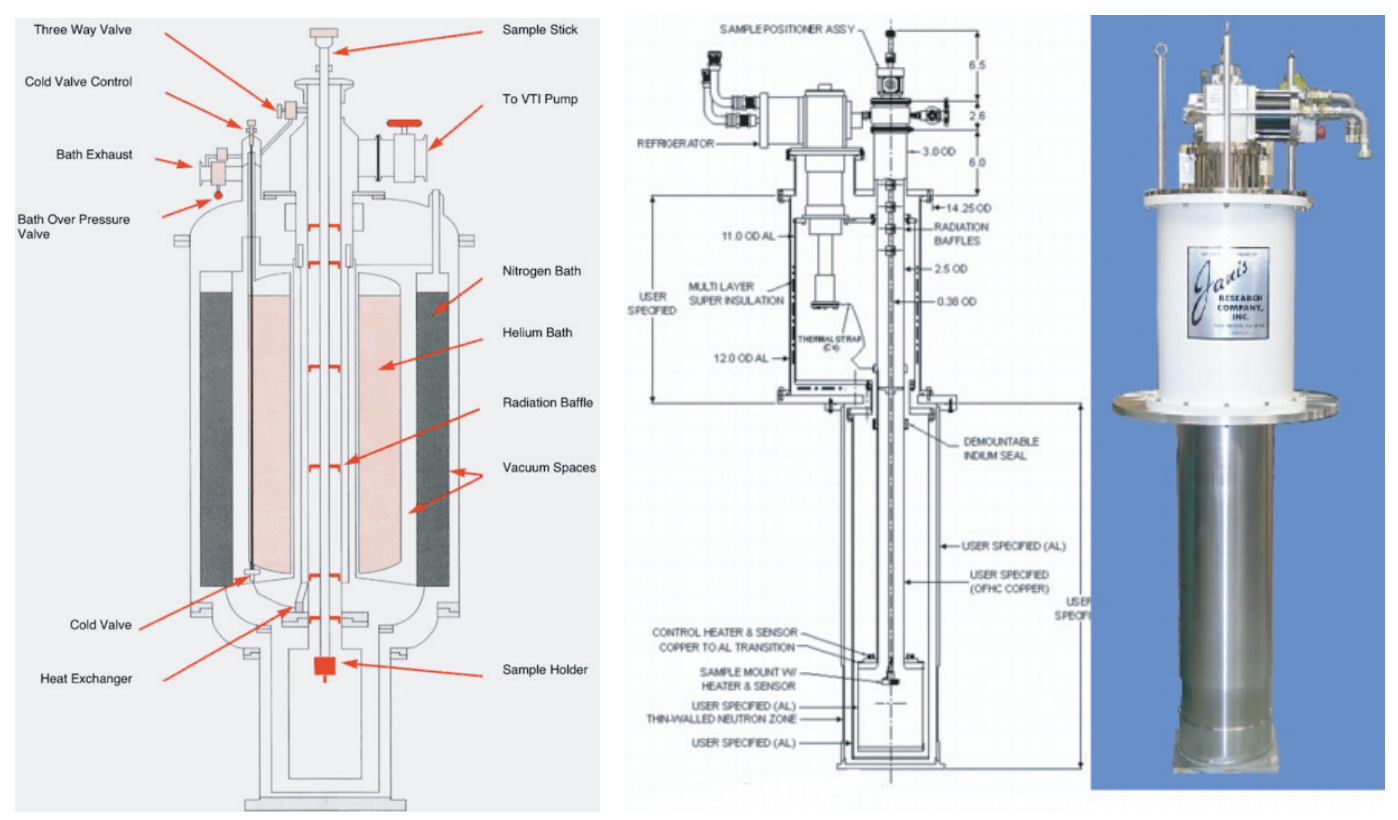

Figure 2. À gauche : vue en coupe d'un cryostat orange (AS Scientific Products Ltd.); à droite : cryogénérateur Gifford-MacMahon à deux étages pour diffusion de neutrons (Janis Research Company, Inc.). 
atteindre environ $10 \mathrm{~K}$, ou à deux étages avec des températures limites proches de $3 \mathrm{~K}$ et une puissance frigorifique à $4 \mathrm{~K}$ de l'ordre du watt (Fig. 2, à droite). Les réfrigérateurs GMM présentent l'avantage de pouvoir fonctionner dans toutes les orientations et se prêtent donc bien aux mesures sur les diffractométres quatre-cercles. Les systèmes dits "à tube pulsé", voisins des précèdents dans leur principe mais ne possédant pas de piston mobile, offrent des performances similaires avec un moindre niveau de vibration à froid ainsi qu'une maintenance réduite. Ils doivent par contre être utilisés dans une orientation proche de la verticale. Tous ces cryogénérateurs sont en pratique intégrés soit dans de simples cryostats à température variable, soit dans des ensembles plus complexes comprenant par exemple un cryoaimant (Section 4). Ils peuvent aussi servir de source de froid primaire dans certains des systèmes que nous allons examiner maintenant, et qui sont destinés à explorer une gamme de températures s'étendant bien au-dessous du degré Kelvin.

\subsection{Au-dessous de $1 \mathrm{~K}$ : réfrigérateurs à dilution}

Les principales méthodes de refroidissement permettant d'atteindre les très basses ou ultra-basses températures (cryostats à ${ }^{3} \mathrm{He}$, réfrigérateurs à dilution ${ }^{3} \mathrm{He}-{ }^{4} \mathrm{He}$, désaimantation nucléaire) ont fait l'objet de nombreuses théses et articles spécialisés, dont on peut trouver les références dans l'ouvrage de référence de Pobell sur le sujet [34]. Pour les mesures de neutrons, la technique la plus utilisée fait appel à des réfrigérateurs à dilution, seuls capables d'assurer une production de froid en continu à des températures inférieures à $0,3 \mathrm{~K}$. Différents fabricants proposent aujourd'hui tant des systèmes autonomes que des "inserts" conçus pour s'adapter à un cryostat classique (Figure 3), à flux d'hélium ou (plus récemment) à cryogénérateur, lequel fournit la puissance frigorifique nécessaire au refroidissement et à la condensation du mélange injecté depuis la température ambiante. Ces dispositifs utilisent l'absorption de chaleur associée au mélange des deux isotopes ${ }^{3} \mathrm{He}$ et ${ }^{4} \mathrm{He}$ de l'hélium au-dessous de la température de "séparation de phase" proche de $0,8 \mathrm{~K}$. Le froid est produit dans une "boîte à mélange" contenant les deux phases liquides, l'une concentrée $\left(x_{3} \approx 1\right)$ et l'autre diluée $\left(x_{3} \approx 0,065\right.$ pour $T \rightarrow 0$ ), lorsque des atomes d ${ }^{3} \mathrm{He}$ sont transférés de la première phase vers la seconde. La puissance
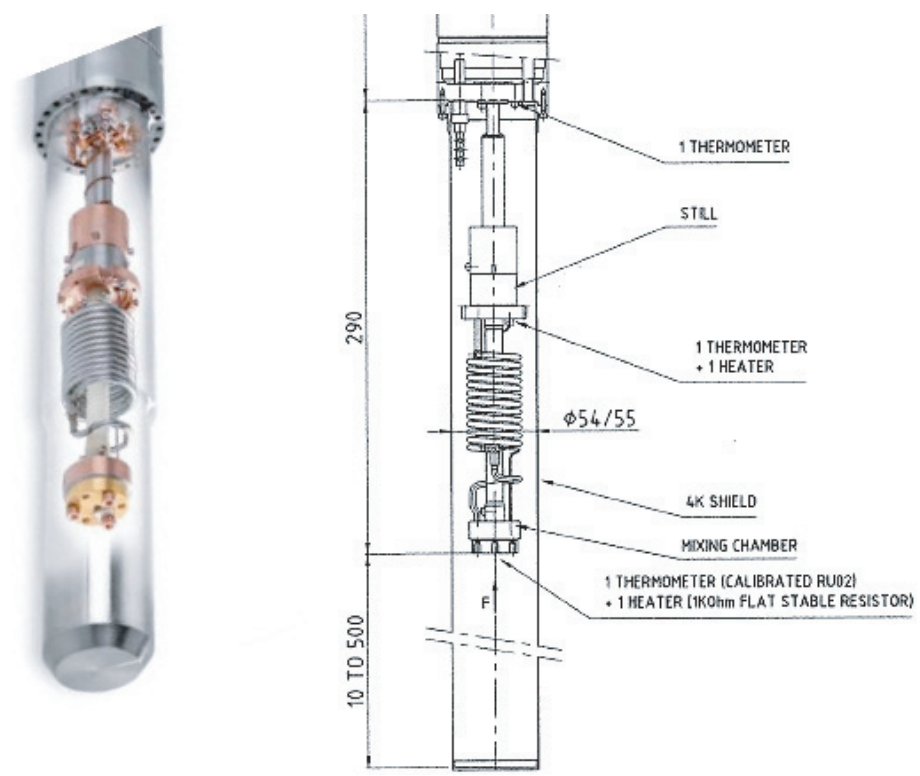

Figure 3. Insert à dilution ${ }^{3} \mathrm{He}-{ }^{4} \mathrm{He}$ (Air Liquide/DTA). 
frigorifique (en watt) est donnée par l'expression $\dot{Q}=84 \dot{n}_{3} T^{2}$, oú $\dot{n}_{3}$ est le débit de circulation d'hélium 3 en mole/s et $T$ la température en kelvin.

Dans l'environnement des sources de neutrons, on trouve maintenant couramment des appareillages ayant une température de base proche de 50 ou $100 \mathrm{mK}$ avec une puissance de l'ordre de $100 \mu \mathrm{W}$, et capables de refroidir non seulement les échantillons mais aussi, selon les besoins, d'autres dispositifs tels que des chambres de pression (Section 5.4). Les principales limitations proviennent des apports de chaleur dus aux vibrations ou aux mouvements du spectromètre, ainsi qu'à l'énergie libérée lors de l'absorption de neutrons par l'échantillon lui-même ou son environnement immédiat (l'usage de "masques" en Cd sera donc autant que possible à éviter). Les conditions nécessaires au bon fonctionnement du circuit de dilution excluent en principe d'incliner ce type d'appareil de plus d'une dizaine de degré par rapport à la verticale. Cependant, une solution élégante a été imaginée par Benoît et Pujol [4,5], au départ pour résoudre le problème du fonctionnement en apesanteur d'un réfrigérateur à dilution destiné à refroidir des détecteurs à l'intérieur d'un satellite. Un dispositif fonctionnant sur le même principe (circulation de l'hélium 3 assurée par une "pompe à vortex", permettant de s'affranchir de l'évaporateur, et non-recyclage de l'hélium 3 in situ) a ensuite été développé spécialement pour les mesures sur quatre-cercles. Une version capable d'atteindre une température minimale de $250 \mathrm{mK}$ est en service à l'ILL pour des mesures de diffraction sur monocristal, notamment sur l'instrument D10.

\subsection{Ultra-basses températures : exemples d'applications}

Au-dessous de 30-50 mK, les contraintes de construction et d'exploitation des systèmes cryogéniques augmentent rapidement, et les expériences sortent du cadre des moyens mis à la disposition des utilisateurs par les centres de neutrons. Mais cette limite pratique est loin de constituer l'horizon ultime des mesures de neutrons aux ultra-basses températures, pour autant qu'on soit prêt à y investir le temps et les efforts nécessaires. Parmi les expériences "pionnières" dans ce domaine, on citera la série d'études sur l'ordre nucléaire dans les métaux nobles réalisée par une équipe de l'Université d'Helsinki en liaison avec des neutroniciens du HMI Berlin et du Laboratoire National de Risø (Danemark) $[18,40]$. Dans le cas de l'argent, la température d'ordre nucléaire est inférieure au nanokelvin. Un étage de désaimantation nucléaire de cuivre équipé d'une bobine de 9 teslas est utilisé pour pré-refroidir l'échantillon de ${ }^{109} \mathrm{Ag}$, lui-même maintenu sous un champ constant de $7 \mathrm{~T}$, jusqu'à $0.1 \mathrm{mK}$. Cette étape est suivie d'une désaimantation de l'échantillon dans laquelle le système des spins nucléaires de l'argent se refroidit jusqu'à quelques centaines de picokelvin, alors que le réseau et les électrons demeurent en contact thermique avec le réfrigérant de cuivre. Ce découplage est rendu possible par le temps de relaxation spin-réseau qui est ici de l'ordre de 10-20h. Le système nucléaire se réchauffe ensuite en quelques heures, principalement sous l'effet du réchauffement par le faisceau de neutrons. La polarisation nucléaire et la température de spin sont déduites d'une mesure de transmission en neutrons polarisés. Les résultats (Fig. 4) démontrent l'existence d'un ordre à longue distance des spins nucléaires $I=\frac{1}{2}$ avec un vecteur d'onde $\boldsymbol{k}=(0,0,1)$, au-dessous d'une température d'ordre estimée de $700 \pm 80 \mathrm{pK}$.

D'autres études de diffraction ont été effectuées, à des températures plus proches du millikelvin, par exemple sur l'ordre nucléaire de l'holmium dans le composé de Van Vleck à renforcement hyperfin $\mathrm{HoVO}_{4}$, là encore auto-refroidi par désaimantation nucléaire [38]. Signalons enfin la mise en évidence [3] de la structure nucléaire antiferromagnétique (AF) de l'hélium 3 solide, performance réalisée dés 1985 sur la modeste source Mélusine (aujourd'hui fermée) du CEA/Grenoble et restée à ce jour inégalée. La mise en place en 2000 d'un réseau européen intitulé Magnetic properties of ${ }^{3} \mathrm{He}$ by means of neutron diffraction marque une reprise de l'activité sur cette thématique, avec l'objectif ambitieux d'étudier la structure nucléaire AF dans les deux phases ordonnées à basse pression en champ nul et sous champ puis, sous une pression supérieure à $10 \mathrm{MPa}$, de tenter de mettre en évidence un ordre ferromagnétique prévu par la théorie, cette fois au-dessous de $20 \mu \mathrm{K}$. Ces études sont importantes 


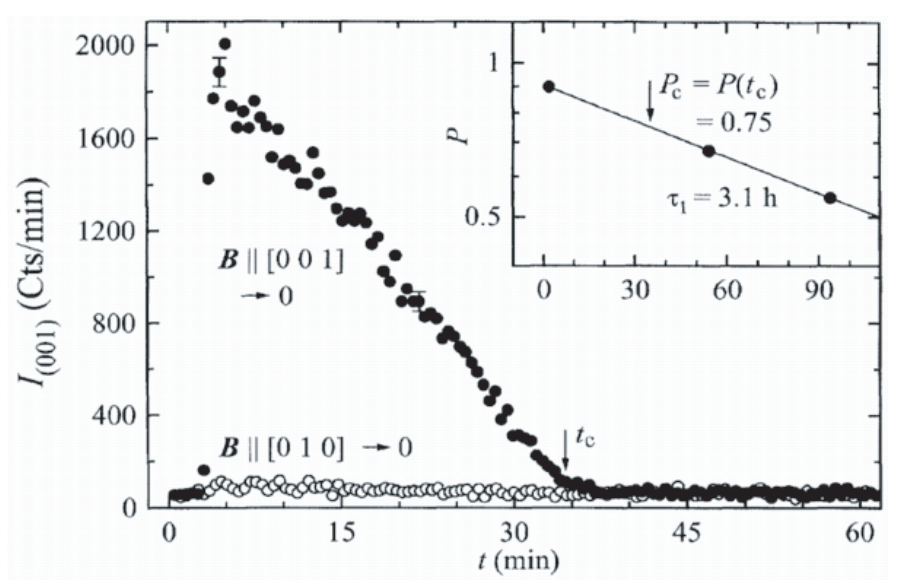

Figure 4. Décroissance en champ nul de l'intensité du pic de Bragg 001 associé à l'ordre nucléaire antiferromagnétique dans ${ }^{109} \mathrm{Ag}$. Le pic n'est observé que si le champ appliqué lors de l'apparition de l'ordre est orienté suivant la direction [ $\left[\begin{array}{lll}0 & 0 & 1\end{array}\right]$, ce qui reflète la population des domaines magnétiques. La température d'ordre est déduite de la polarisation des moments dans l'état paramagnétique, par interpolation sur la courbe de décroissance exponentielle tracée dans l'encart (d'après la référence [40]).

pour une meilleure compréhension des mécanisme d'échange à plusieurs particules responsables des couplages magnétiques dans ce système.

Dans la pratique, on assiste actuellement à une banalisation croissante des mesures jusque vers $50 \mathrm{mK}$, les fabricants s'efforçant de produire des matériels de plus en plus robustes et automatisés. Une des tendances est l'utilisation des cryogénérateurs pour réaliser des systèmes sans azote ni hélium liquides (hormis bien sûr le mélange ${ }^{3} \mathrm{He}-{ }^{4} \mathrm{He}$ ). Un autre point important est de mieux couvrir la zone de raccordement, autour de $1 \mathrm{~K}$, entre les cryostats classiques et les réfrigérateurs à dilution, oú ces derniers sont intrinséquement sujets à de fortes instabilités de température. Enfin, la demande s'oriente de plus en plus vers une combinaison de conditions extrêmes associant les très basses températures avec notamment les forts champs magnétiques et les très hautes pressions.

\section{FORTS CHAMPS MAGNÉTIQUES}

Un des principaux atouts de la diffusion des neutrons étant leur interaction avec le champ magnétique des électrons non appariés, on conçoit bien que les études de magnétisme y occupent une place considérable, et que la possibilité d'appliquer un fort champ magnétique sur l'échantillon soit de ce fait vitale pour bon nombre d'expériences. On ne s'étendra pas ici sur les électroaimants classiques. Leur gamme de champ accessible n'excède guère les $2 \mathrm{~T}$, et les appareillages produisant plus de $1 \mathrm{~T}$ sont assez volumineux (Fig. 5). Dans tous les cas, un refroidissement par eau est nécessaire. Ces équipements restent cependant d'un usage assez courant du fait de leur simplicité de mise en œuvre et de la latitude qu'ils offrent dans l'orientation du champ magnétique.

Pour toutes les études nécessitant des champs supérieurs, l'emploi des cryoaimants supraconducteurs s'est généralisé sur les sources de neutrons. On y utilise comme ailleurs des bobinages fabriqués, selon la gamme de champ, en $\mathrm{NbTi}$ ou en $\mathrm{Nb}_{3} \mathrm{Sn}$ et généralement immergés dans de l'hélium liquide à $4.2 \mathrm{~K}$ ou à $2.3 \mathrm{~K}$ (systèmes dits à "plaque lambda" ou à "bain Claudet" fonctionnant à la température de superfluidité de l'hélium). Depuis quelques années, certains constructeurs proposent aussi des cryoaimants dont les enroulements sont refroidis au moyen d'un cryogénérateur de forte puissance mais ce type d'appareillage est encore assez peu répandu, en particulier pour les très hautes valeurs du champ. Dans la conception classique, l'hélium liquide contenu dans le réservoir principal 


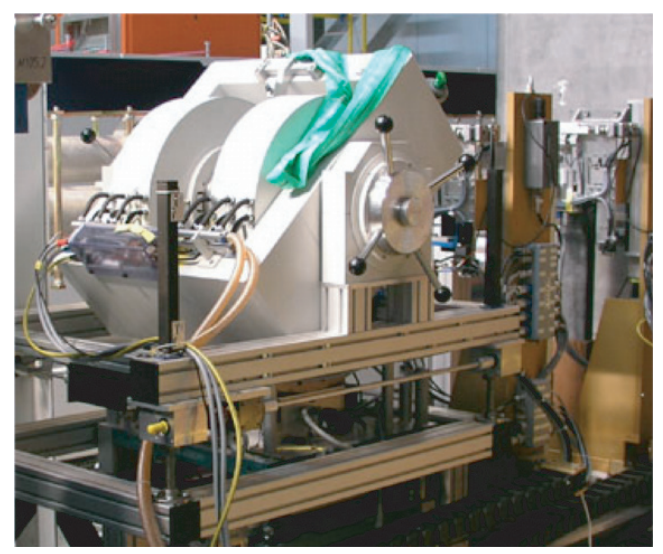

Figure 5. Électroaimant Brucker de 1 tesla installé sur le réflectométre de neutrons AMOR à l'Institut Paul Scherrer, Villigen (Suisse).

sert à la fois à refroidir les bobines et à alimenter un circuit fournissant la puissance frigorifique au "VTI" (insert à température variable) dans lequel se trouve l'échantillon. Le fonctionnement est à cet égard analogue à celui d'un cryostat orange. Dans tous les cas, l'obligation de ménager un passage au faisceau de neutrons impose de recourir à des solutions techniques particulières. On distinguera deux types de dispositifs selon que le champ y est appliqué verticalement ou horizontalement.

\subsection{Cryoaimants à champ vertical}

Pour cette orientation, la géométrie utilisée est celle des bobines de Helmholtz (en anglais : "split pairs") d'axe vertical (Fig. 6). Une des principales difficultés vient des forces magnétiques considérables qui s'exercent entre les deux enroulements aux grandes valeurs du champ. Pour assurer la tenue mécanique de l'ensemble, on aura recours soit à un jeu d'entretoises annulaires en alliage d'aluminium si l'on souhaite privilégier l'invariance par rotation de façon à garantir que l'épaisseur et la nature des matériaux traversés soient les mêmes quelle que soit la direction des neutrons incidents et diffusés (Fig. 6(c)), soit au contraire des “piliers" à 120 ou 180 degrés si l'on préfère minimiser l'épaisseur de matière à traverser en s'accommodant de l'existence d'angles morts. Un autre aspect important dans la conception des bobinages est la valeur de l'ouverture angulaire verticale, qui résultera le plus souvent d'un compromis entre le champ maximum désiré et l'angle solide couvert par les détecteurs, en tenant compte également de son incidence sur la valeur des champs de fuite. Dans les expériences de diffraction sur monocristal utilisant un détecteur à "bras levant", on aura ainsi intérêt à adopter une ouverture asymétrique (par exemple de $-5^{\circ}$ à $+20^{\circ}$ ) afin de pouvoir atteindre un maximum de réflexions distinctes en dehors du plan horizontal. En outre, une géométrie asymétrique est indispensable dans le cas des neutrons polarisés afin d'éviter que le champ ne s'annule en un point sur le trajet du faisceau, entraînant un risque de dépolarisation. Dans tous les cas, la mise en œuvre de champs de cet ordre dans l'environnement d'une expérience de neutrons impose de s'assurer que les forces exercées sur d'éventuels éléments magnétiques (modules du spectromètre, protections, équipements annexes) et, par réaction, sur les bobines elles-mêmes, ne risquent pas d'occasionner des dégâts matériels, voire des accidents plus sérieux. Il convient pour ce faire de recourir au maximum à des composants amagnétiques.

En ce qui concerne les performances, on gardera à l'esprit qu'en géométrie de Helmholtz, le champ auquel sont soumis les conducteurs dépasse sensiblement celui qui est vu par l'échantillon placé dans l'intervalle entre les bobinages. Il en résulte donc nécessairement une perte de performance par rapport à un solénoïde de conception équivalente. À l'heure actuelle, on obtient assez facilement des champs 

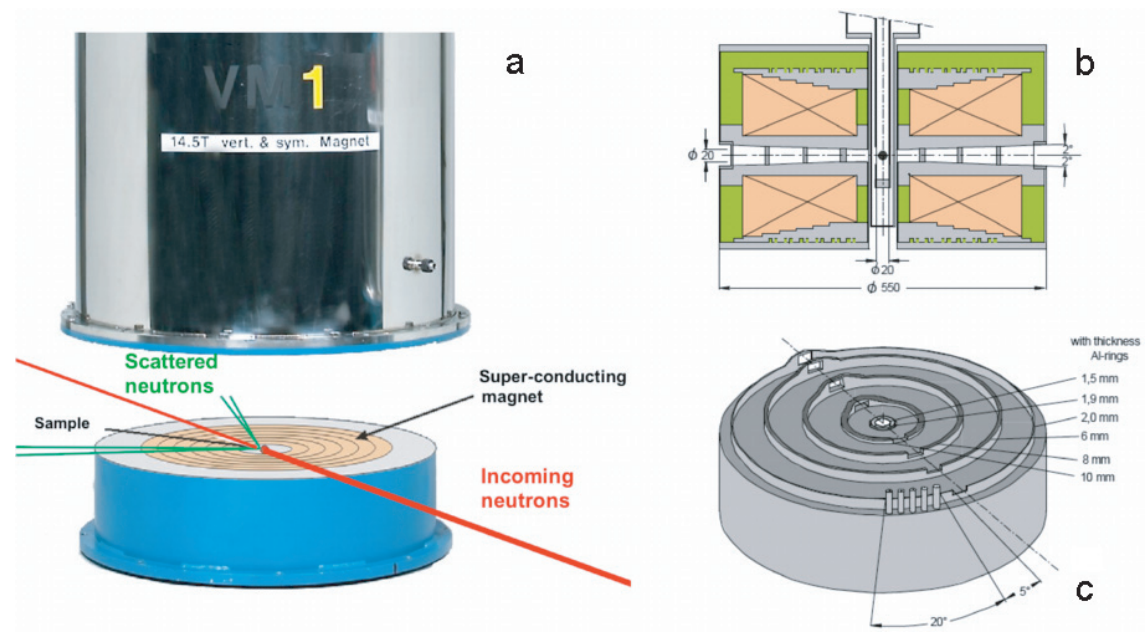

Figure 6. Cryoaimant Oxford Instruments à champ vertical de $15 \mathrm{~T}$ en service au HMI Berlin. (a) Vue éclatée et trajet du faisceau ; (b) coupe des bobines de Helmholtz ; (c) schéma montrant la disposition des entretoises. (Extrait du U.S.E. Handbook du HMI.)

de l'ordre de 8 à 10 T. Au-delà, les coûts augmentent rapidement, de même que les contraintes de mise en œuvre liées à l'importance des champs de fuite qui imposent de travailler dans un environnement pratiquement amagnétique. Malgré ces contraintes, des systèmes atteignant 14,5-15 T ont été réalisés par la société Oxford Instruments et fonctionnent dans plusieurs centres de neutrons (HMI Berlin [28], ILL Grenoble, PSI Villigen, FRM-II Münich). Signalons que l'appareillage du HMI dispose en option d'un système concentrateur de flux constitué de deux éléments en dysprosium polycristallin texturé qui permet de produire, dans un espace plus restreint, un champ allant jusqu'à $17 \mathrm{~T}$.

\subsection{Cryoaimants à champ horizontal}

Pour certaines mesures de diffusion inélastique sur des systèmes magnétiques, il peut être avantageux d'aligner les moments magnétiques suivant la direction du vecteur de diffusion $\boldsymbol{Q}$ afin que les deux composantes transverses des excitations lui soient perpendiculaires et contribuent ainsi à l'intensité mesurée. Si, comme sur les spectromètres trois-axes, le vecteur $\boldsymbol{Q}$ est confiné à l'intérieur du plan horizontal, il devra en être de même du champ magnétique appliqué. À première vue, cette géométrie peut sembler favorable puisqu'il va être possible d'orienter un solénoïde d'axe horizontal dans une géométrie compatible avec le passage du faisceau de neutrons, au moins pour des angles de diffusion pas trop grands. En pratique cependant, on aura le plus souvent besoin d'un accés au centre de la bobine suivant l'axe vertical, ne serait-ce que pour permettre l'introduction d'une enceinte à température variable (cryogénérateur, cryostat, etc.). On en revient alors au cas des bobines d'Helmholtz, mais avec leur axe orienté cette fois suivant l'horizontale. Il est clair que cette conception comporte de sérieuses limitations quant aux angles de diffusion accessibles, et supposera donc un certain compromis entre la valeur maximale du champ (de l'ordre de $6 \mathrm{~T}$ ) et les ouvertures angulaires. Une autre approche consiste à utiliser non plus deux mais quatre bobines de sorte que tous les enroulements puissent se trouver en dehors du plan horizontal. Le principe de ce dispositif est indiqué sur la Figure 7 représentant un aimant Oxford Instruments de $4 \mathrm{~T}$, dont des exemplaires sont en service à l'ILL et au HMI. Les angles morts sont ramenés à deux fois $20^{\circ}$, mais au prix d'une construction sensiblement plus complexe, dont l'incidence sur le coût est loin d'être négligeable. 

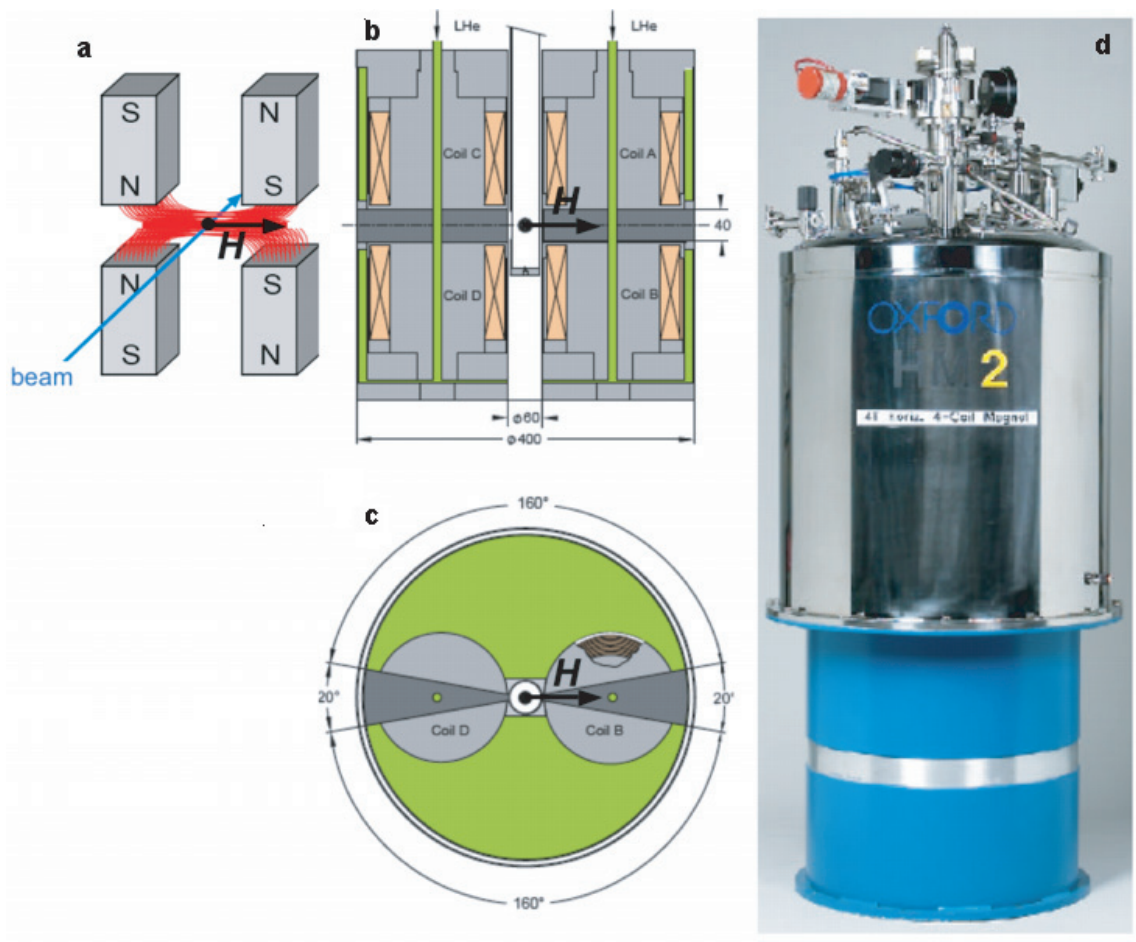

Figure 7. Cryoaimant Oxford Instruments à champ horizontal de $4 \mathrm{~T}$ en service au HMI Berlin. (a) schéma de principe ; (b) coupe des bobines de Helmholtz ; (c) angles d'ouverture dans le plan horizontal ; (d) vue d'ensemble. (Extrait du U.S.E. Handbook du HMI.)

\section{3 Études de diffusion élastique ou inélastique en fort champ}

Les dispositifs qui précèdent fonctionnant de maniére régulière sur différentes sources de neutrons, les études réalisées sont évidemment nombreuses et couvrent de multiples aspects des phénomènes magnétiques dans les solides. Les deux exemples ci-dessous sont empruntés à des études récentes sur les systèmes à ordre multipolaires et sur les systèmes de spins quantiques de basse dimensionnalité.

$\mathrm{PrOs}_{4} \mathrm{Sb}_{12}$ est le premier composé connu à base de praséodyme présentant une supraconductivité de type "fermions lourds", et certains ont vu dans cette supraconductivité la conséquence de fluctuations quadrupolaires. Dans ce cadre, l'existence à basse température d'une phase ordonnée induite sous champ magnétique a suscité un vif intérêt. Les résultats de Kaneko et collab. [19] (Fig. 8), obtenus par diffusion élastique de neutrons sur le spectromètre LTAS du Japan Atomic Energy Research Institute (JAERI) à Tokai, ont nécessité l'application de champs de $10 \mathrm{~T}$ à des températures de l'ordre de $200 \mathrm{mK}$. Ils montrent l'existence, dans la nouvelle phase (notée "A" sur le diagramme de phase de la Figure 8 [39]), d'un faible pic magnétique de surstructure associé au vecteur d'onde $\boldsymbol{k}=(1,0,0)$ pour un champ orienté suivant la direction [110]. Ce résultat ne peut s'expliquer par un couplage purement magnétique et traduit l'existence d'un ordre sous-jacent des moments quadrupolaires de symétrie $O_{x y}$ du praséodyme. Il va donc dans le sens d'un rôle possible joué par les interactions quadrupolaires dans la supraconductivité de ce composé.

Les champs forts jouent aussi un rôle essentiel pour l'étude des excitations dans les systèmes magnétiques à fluctuations quantiques. L'exemple choisi est tiré d'expériences de diffusion inélastique sur monocristal réalisées à HMI sur un spectromètre trois-axes [11]. Le composé $\mathrm{CuGeO}_{3}$ est un système modéle de spins $1 / 2\left(\mathrm{Cu}^{2+}\right)$ à interactions $\mathrm{AF} 1 \mathrm{D}$ de type Heisenberg. Il présente en champ 

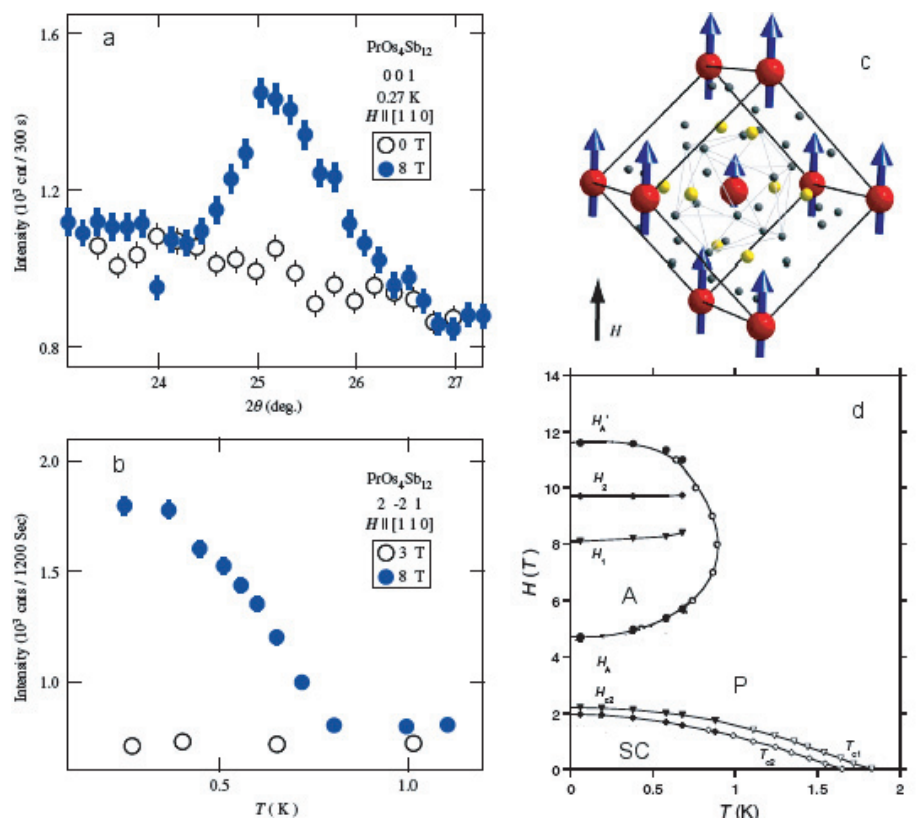

Figure 8. Mise en évidence (a) et dépendance en température (b) du pic de Bragg 001 caractéristique de la composante antiferromagnétique (c) induite sous champ $\left(H \|[110]\right.$ ) dans $\mathrm{PrOs}_{4} \mathrm{Sb}_{12}$ (région notée A dans le diagramme de phase du cadre (d). (D'après les références [19] (a)-(c) et [39] (d).)
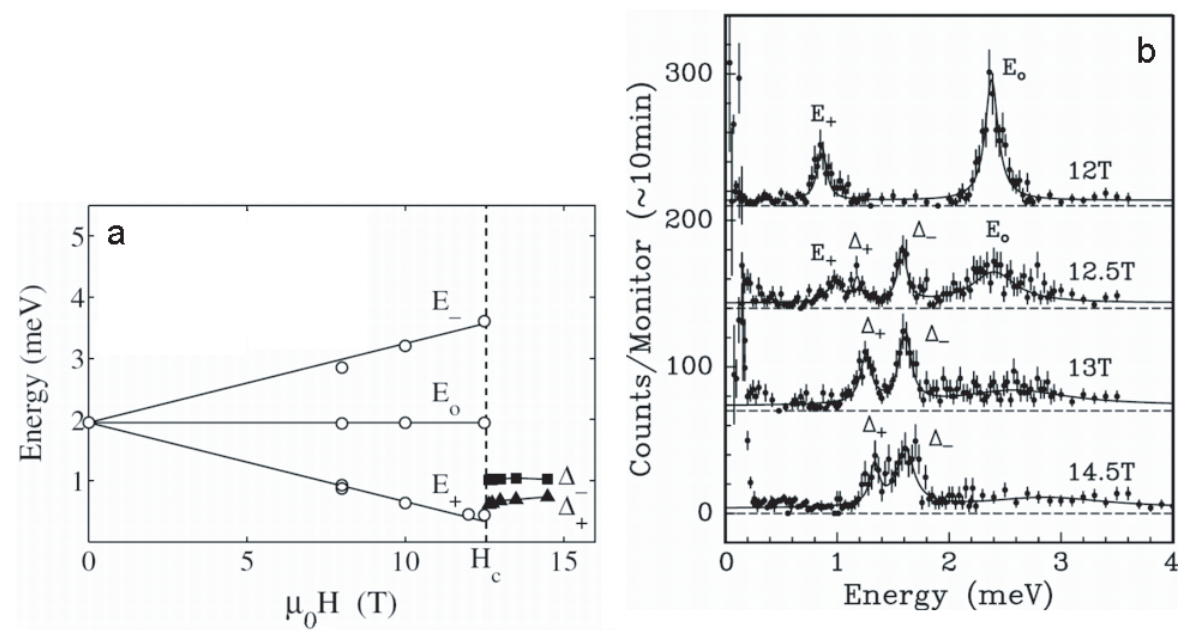

Figure 9. Excitations magnétiques dans les phases dimérisée et incommensurable du composé spin-Peierls $\mathrm{CuGeO}_{3}$ sous champ magnétique à $T=2 \mathrm{~K}$. (a) : $\mathbf{Q}=\left(0,1, \frac{1}{2}\right) ;(\mathrm{b}): \mathbf{Q}=\left(\frac{1}{2}, 1, \frac{1}{2}\right)$ (d'après la référence [11]).

nul un état fondamental dimérisé "spin-Peierls" avec un triplet d'excitations de basse énergie $\left(S_{\text {tot }}=1\right)$ correspondant à la formation de paires de parois de domaines encore appelées "solitons". Sous l'effet d'un champ, ce triplet se scinde en trois niveaux (Fig. 9(a)), dont les deux plus bas, $E_{+}$et $E_{0}$ sont bien visibles sur le spectre à $H=12 \mathrm{~T}$ (Fig. 9(b), en haut). Au-dessus de $H_{c}=12,58 \mathrm{~T}$, alors que l'énergie du niveau le plus bas approche de zéro, le système subit une transition du premier ordre vers un nouvel état oú les parois de domaines sont gelées par les interactions élastiques tridimensionnelles dans une 


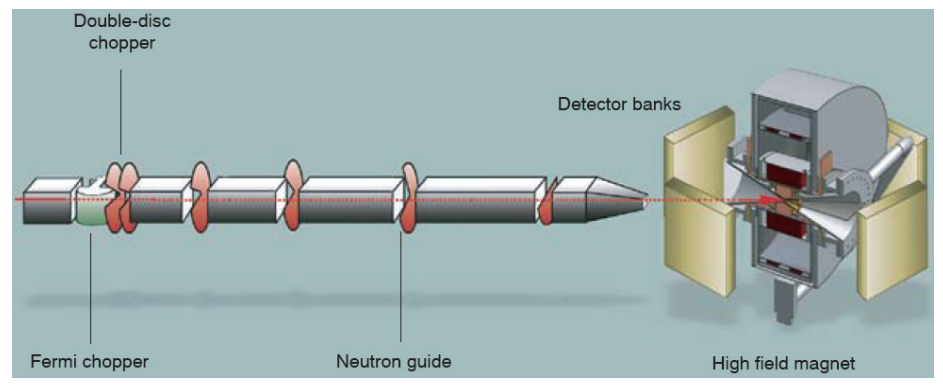

Figure 10. Projet d'aimant hybride de $30 \mathrm{~T}$ au HMI Berlin : schéma de l'implantation sur le guide thermique et vue éclatée des bobines (extrait de la référence [6]).

structure statique incommensurable. Deux nouvelles excitations $\Delta_{+}$et $\Delta_{-}$apparaissent au voisinage du centre de zone $\operatorname{AF}(0,1,1 / 2)$ (Fig. 9(b)). Elles sont interprétées, en accord avec des modéles théoriques de la phase spin-Peierls IC, comme des modes transverses associés à la création ou à l'annihilation d'une paire de solitons.

\subsection{Perspectives pour des champs supérieurs à $20 \mathrm{~T}$}

Dans le cadre des connaissances actuelles, les cryoaimants supraconducteurs décrits aux paragraphes 4.1 et 4.2 semblent près d'atteindre leurs limites. Pour aller au-delà, une voie prometteuse consiste à utiliser une technologie hybride associant un cryoaimant supraconducteur refroidi à l'hélium liquide et un insert "résistif" en cuivre. Le projet le plus avancé est celui développé au HMI, qui vise à terme l'obtention d'un champ maximum de l'ordre de $30 \mathrm{~T}$ pour une puissance électrique de $8 \mathrm{MW}$. La formule retenue, mise au point au National High Magnetic Field Laboratory de Tallahasee (USA), présente la particularité d'alimenter en série l'insert en cuivre (de type dit "Florida-Bitter") et les bobines supraconductrices à partir d'une même source pouvant délivrer un courant de $20000 \mathrm{~A}$. Les enroulements de $\mathrm{Nb}_{3} \mathrm{Sn}$ feront appel à une technologie CICC (de l'anglais "cable-in-conduit conductor") oú les conducteurs (des brins composites cuivre-supra) gainés dans un conduit métallique sont refroidis par de l'hélium circulant à l'intérieur de ce dernier. L'axe du champ sera orienté dans le plan horizontal et l'intérieur de la bobine, de forme conique, offrira une ouverture totale de l'ordre de $30^{\circ}$. L'ensemble fera partie intégrante du nouveau spectromètre ExED (Extreme environment spectrometer) qui sera installé sur un nouveau guide de neutrons thermiques à supermiroirs (Fig. 10). L'option retenue est celle de mesures en temps de vol pour la diffusion élastique comme inélastique en raison des contraintes imposées par la géométrie de la bobine. Le développement d'environnements d'échantillons adaptés devra également composer avec cette limitation.

Un projet similaire, pouvant être associé ou non à une seconde bobine de type Helmholtz à champ vertical, a été proposé dans le cadre du développement d'une ligne "haut champ" pour la nouvelle source de neutrons pulsée SNS d'Oak Ridge.

\subsection{L'option des champs pulsés}

Si l'on prend en considération à la fois les limites intrinséques des matériaux supraconducteurs et les puissances électriques prohibitives exigées par les bobines résistives (40 MW dans une première version du projet HMI conçue pour $40 \mathrm{~T}$ ), la seule issue possible pour produire des champs encore plus intenses semble être l'utilisation de systèmes pulsés. Si différents laboratoires dans le monde, dont le Laboratoire National des Champs Magnétiques Pulsés de Toulouse, ont une expertise reconnue dans cette technique, l'application aux mesures de neutrons se limite pratiquement au travail de deux équipes, l'une russe au Joint Institute for Nuclear Research (JINR) de Dubna [12], l'autre japonaise au National Laboratory 

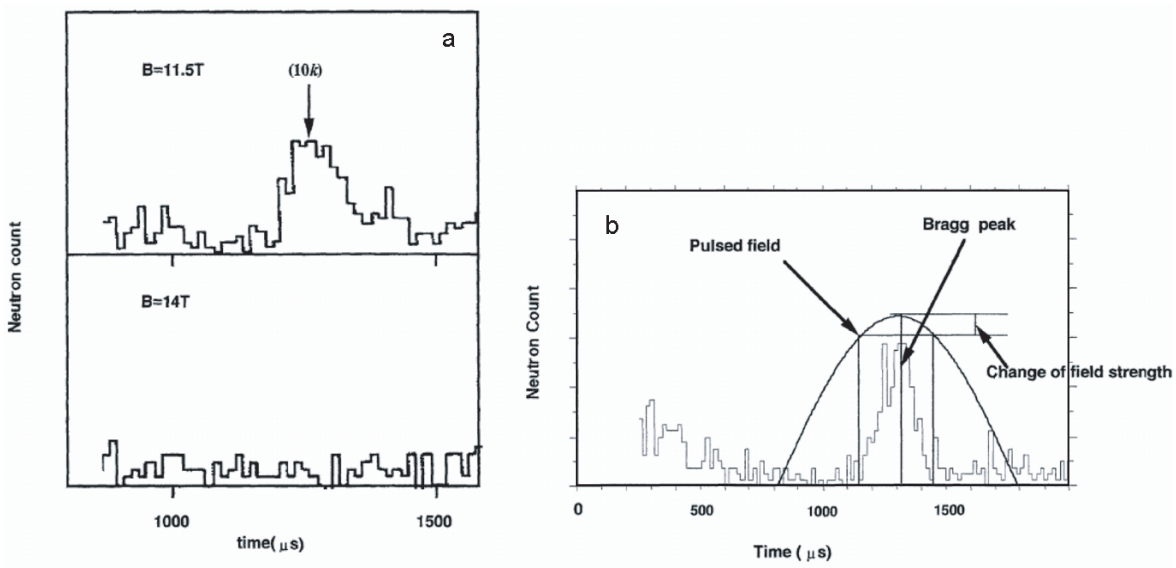

Figure 11. Dépendance en champ du pic de Bragg incommensurable $\left(1,0, k^{\prime}\right)$ dans $\operatorname{PrCo}_{2} \mathrm{Si}_{2} ;$; (a) signal mesuré en champ magnétique pulsé ; (b) profil de l'impulsion de champ. (D'après la référence [32].)

for High Energy Physics (KEK) à Tsukuba [32]. Dans ces appareillages, le champ est produit par des bobines dont la conception est dérivée de celle de Bitter, alimentées par la décharge d'une batterie de condensateurs. La durée des impulsions est de l'ordre de la milliseconde et leur période se situe autour de la seconde. Dans les deux cas, la source de neutrons elle-même est de type pulsé (à fission pour le réacteur IBR-2 de Dubna, à spallation pour la source de Tsukuba). L'impulsion de champ est synchronisée avec celle des neutrons mais sa fréquence de répétition est moindre. La conception des bobines doit naturellement prendre en compte les contraintes mécaniques et thermiques considérables qu'elles subissent lors de la décharge, par exemple en remplaçant le cuivre des bobines classiques par un alliage $\mathrm{Cu}-\mathrm{Be}$. Même ainsi, la durée de vie des bobines reste un paramètre limitant dans la définition du mode d'utilisation.

La Figure 11 présente un exemple de mesure de diffraction réalisée sur le diffractométre MRP de Tsukuba par une méthode de temps de vol [32]. Le composé $\mathrm{PrCo}_{2} \mathrm{Si}_{2}$ présente plusieurs phases magnétiques successives et le tracé traduit la disparition du pic de Bragg incommensurable en (1 0 0,225 ) entre $H=11,5$ et $14 \mathrm{~T}$ marquant la transition vers un état ferromagnétique. Le cadre (b) montre que la valeur du champ reste constante à mieux de $10 \%$ prés sur une durée correspondant à la largeur du pic.

L'installation sur laquelle ces expériences ont été réalisées est aujourd'hui arrêtée. Elle doit être remplacée par un nouvel instrument beaucoup plus performant qui sera implanté sur la source J-PARC de Tokai. Il comportera une batterie de condensateurs de $250 \mathrm{~kJ}$ pouvant alimenter soit une bobine de Helmholtz de 40-45 T, soit un solénoïde de $60 \mathrm{~T}$. La durée prévue des impulsions est de $10 \mathrm{~ms}$ avec, aux valeurs extrêmes du champ, un délai d'une quinzaine de minutes entre les "tirs". Lorsque la source fonctionnera à sa puissance maximale, la mesure d'un pic de diffraction ne devrait nécessiter que quelques "tirs" [33]. Un équipement similaire est en cours de construction sur le réacteur JRR-3M de Tokai pour un champ maximum de 35-40 T.

Dans un article récent [31], Nietz a évoqué la possibilité de produire des champs encore plus élevés (jusqu'à 50, voire $80 \mathrm{~T}$ ) à condition que la mesure puisse être réalisée en une seule impulsion et qu'on s'affranchisse ainsi de la contrainte liée à la durée de vie de la bobine. D'après ses estimations, les flux de neutrons requis pourraient être obtenus sur des "réacteurs pulsés apériodiques" [10] tels que BIGR à Sarov (Russie) dont le flux atteint $2.5 \cdot 10^{18}$ therm. $\mathrm{n} /\left(\mathrm{cm}^{2} \mathrm{~s}\right)$ pendant une impulsion de $2 \mathrm{~ms}$. La seule expérience de ce type rapportée à ce jour a été réalisé sur le petit réacteur TRIGA Mark II de $250 \mathrm{~kW}$ à l'Université de Vienne, dont la puissance peut monter jusqu'à $380 \mathrm{MW}$ durant quelques millisecondes. 
La suppression de l'antiferromagnétisme dans un monocristal de $\mathrm{MnF}_{2}$ y a effectivement été observée au cours d'une impulsion unique jusqu'à un champ d'environ $22 \mathrm{~T}$ [1]. L'avenir de ces techniques de "champs extrêmes" demeure néanmoins assez hypothétique.

\section{HAUTES PRESSIONS}

Les motivations pour recourir aux hautes pressions peuvent être très diverses. On a évoqué dans l'introduction l'aventure scientifique qu'a constitué la liquéfaction successive des gaz sous des pressions de quelques dizaines de bars. Pour les expériences sur l'hélium 3 solide telles que celles du paragraphe 3.2, la croissance du monocristal étudié nécessite de travailler au-dessus de $3 \mathrm{MPa}$, sur la courbe de solidification. Des pressions plus élevées, dans la gamme 0,1-10 GPa, permettent d'explorer les diagrammes de phases cristallins et magnétiques de nombreux composés en faisant varier le volume de leur maille dans des proportions atteignant 10\%. Au-delà, les conditions sont de l'ordre de celles qui régnent à l'intérieur du manteau terrestre (350 GPa au centre de la Terre) et présentent donc un intérêt particulier pour les études de géophysique. Certains sujets plus fondamentaux, tels que la physique des solides quantiques, peuvent également en bénéficier.

Des percées importantes réalisées au cours des quinze derniéres années ont permis de repousser les limites des pressions accessibles aux mesures de neutrons, comme on le verra dans les paragraphes 5.3 et 5.4. Cependant il importe avant tout de choisir pour chaque expérience l'appareillage le mieux adapté non seulement en fonction de la valeur absolue de la pression, mais aussi d'autres facteurs comme l'hydrostaticité, le volume disponible pour l'échantillon, la géométrie de diffusion pour les neutrons, ou encore le bruit de fond généré par l'appareil. Nous commencerons donc par donner un bref aperçu des principales techniques susceptibles d'être utilisées selon la gamme de pression.

\subsection{Les chambres de pression hydrostatiques à hélium}

Ces systèmes se composent de la chambre proprement dite (en alliage d'aluminium, de Ti-Zr, ou de $\mathrm{Cu}-\mathrm{Be}$ ), reliée à un banc à pression d'hélium gazeux par un capillaire à paroi épaisse. La gamme accessible n'excède généralement pas $0,5-0,7 \mathrm{GPa}$, mais le diamétre intérieur utile peut dépasser 10 $\mathrm{mm}$ ce qui est avantageux en particulier pour les mesures de diffusion inélastique nécessitant de gros échantillons. L'utilisation d'alliages d'aluminium permet aussi d'avoir un signal parasite faible et pratiquement isotrope. Surtout, les conditions sont parfaitement hydrostatiques tant que l'hélium est à l'état liquide, et la pression peut être variée in situ. L'étanchéité est généralement assurée par des joints d'étanchéité de type Bridgman ou Paureau. Même dans la phase solide, l'effet des contraintes uniaxiales est très réduit en raison du faible module de cisaillement de l'hélium.

Des précautions particulières doivent être prises pour assurer la sécurité compte tenu de l'énergie élastique importante emmagasinée. Ceci inclut la certification des matériaux constitutifs, l'adaptation d'un pare-éclat autour de la chambre elle-même, l'ancrage de la partie libre du capillaire, et la réalisation d'un test de rupture sur une chambre témoin afin de valider les limites de fonctionnement. Le même type de chambre peut aussi être utilisé pour l'étude de gaz sous haute pression, mais il faut alors veiller à se prémunir contre d'éventuels problèmes de réactivité. Pour plus de détails, on pourra consulter par exemple l'article récent de Kuhs et collab. traitant de ce sujet [25].

\subsection{Les chambres de type piston-cylindre à milieu transmetteur liquide}

Lorsque l'hydrostaticité de la pression est moins critique, ou si l'on souhaite atteindre des pressions plus élevées en s'affranchissant des contraintes liées à l'utilisation d'un compresseur, on peut recourir à des systèmes à milieu transmetteur liquide. Pour les mesures de neutrons, on choisira de préfèrence un liquide non hydrogéné tel que les hydrocarbures perfluorés de la série des Fluorinert (FC-75, mélange FC-84-FC-87 hydrostatique jusque vers 2,3 GPa à l'ambiante $[36,41]$ ), ou des mélanges 


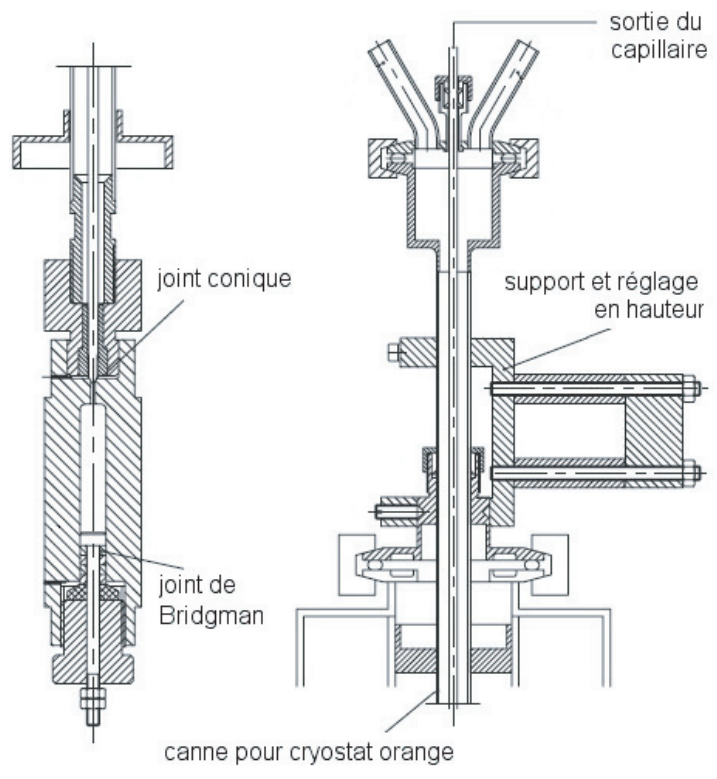

Figure 12. Chambre hydrostatique à hélium en Ti-Zr de l'ILL, avec son adaptation sur un cryostat orange standard (d'après la référence [25]).

d'alcools deutériés (éthanol-méthanol ou pentane-isopentane) restant liquides jusqu'à des pressions supérieures [26].

Avec les chambres de ce type, l'une des principales difficultés est d'optimiser la résistance du cylindre sans trop accroître l'atténuation du faisceau ni les diffusions parasites. On peut pour cela soit utiliser un matériau homogéne ( $\mathrm{Cu}-\mathrm{Be}, \mathrm{Ti}-\mathrm{Zr}$, etc.) présentant les caractéristiques souhaitées, en le renforçant éventuellement par autofrettage, soit opter pour un dispositif du genre de celui développé par McWhan [27] dans lequel le cylindre est fait d'un matériau (en général l'alumine) transparent aux neutrons mais peu résistant en traction, comprimé extérieurement par des coquilles en acier ménageant un espace suffisant pour le passage du faisceau. Des appareils conçus sur ce principe peuvent atteindre des pressions allant jusqu'à $3 \mathrm{GPa}$. Une réalisation récente est la chambre "Kiel-Berlin" représentée sur la Figure 13.

Les chambres destinées aux mesures à basse température sont généralement des "clamps" dont la mise en pression s'effectue sous une presse hydraulique par application d'une force sur l'un des pistons. Ce dernier est ensuite maintenu dans sa position par un écrou de blocage. On dispose alors d'un dispositif indépendant, facile à transporter et prêt à être monté dans l'environnement cryogénique voulu. D'autres appareils, comme celui de la Figure 13, incluent leur propre système hydraulique de pressurisation. Conçue pour les mesures à haute température, cette version est dotée d'un "micro-four" placé à l'intérieur même de la cellule et pouvant atteindre $700 \mathrm{~K}$.

Jusque vers le milieu des années 80 , les possibilités offertes pour la diffusion de neutrons sous haute pression vont pratiquement se limiter à celles qui viennent d'être décrites. Des perspectives intéressantes avaient été ouvertes en Russie par l'utilisation de cellules à enclumes en diamant, mais la taille extrêmement faible des échantillons confinaient cette technique à des cas très particuliers (voir références dans la revue de Somenkov [37]). C'est vers 1990 que le domaine va connaître un nouvel essor sous l'impulsion de deux équipes spécialisées dans les hautes pressions, l'une à l'Institut Kourtchatov de Moscou, l'autre à Paris au Laboratoire de Physique des Milieux Condensés de l'Université Pierre et Marie Curie, en collaboration avec l'Université d'Édimbourg. Menés à partir de deux approches radicalement différentes, leurs travaux vont contribuer à ouvrir de nouveaux champs 

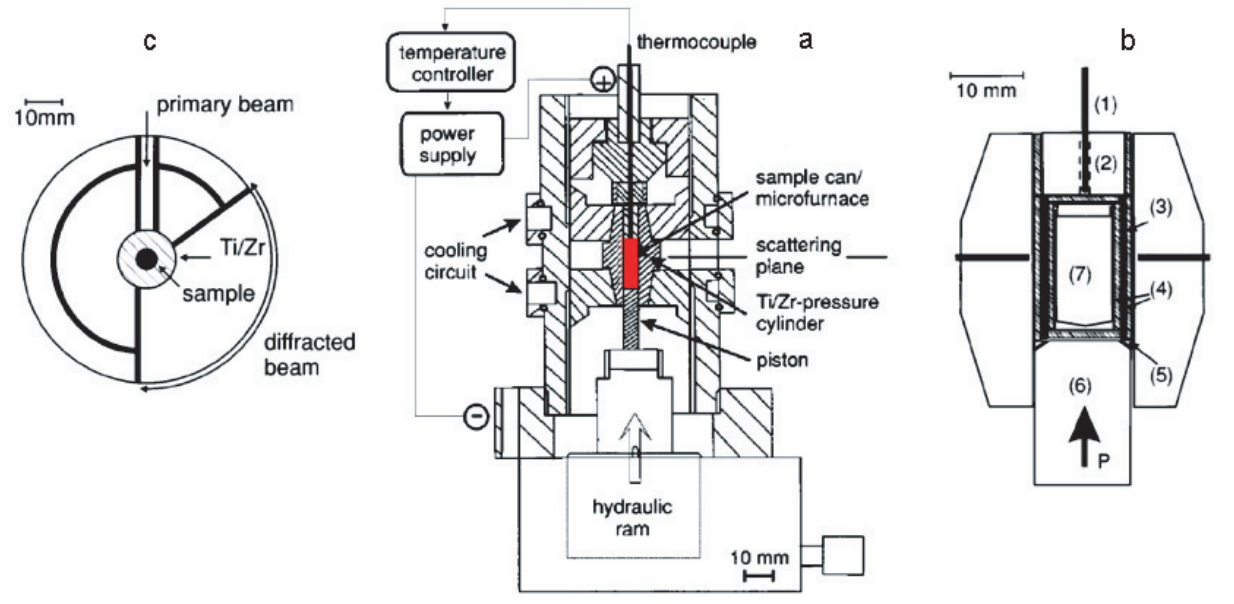

Figure 13. Chambre de pression Kiel-Berlin : (a) schéma d'ensemble avec le système de pressurisation hydraulique ; (b) détail du microfour ; (c) coupe dans le plan de diffusion montrant les angles d'ouverture (d'après la référence [24]).
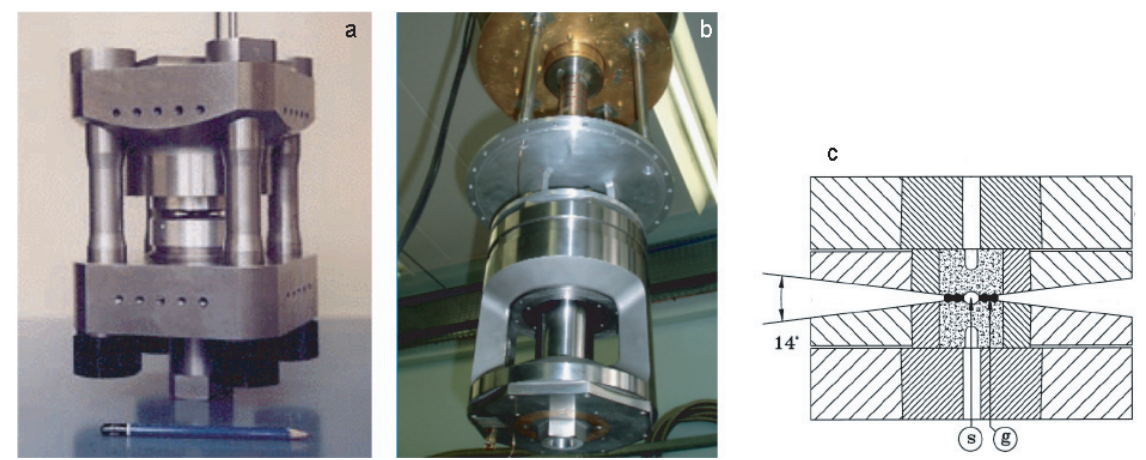

Figure 14. (a) Presse "Paris-Édimbourg" équipée d'une paire d'enclumes toroïdales ; (b) version pour les basses températures montée sur un cryogénérateur à $3 \mathrm{~K}$; (c) coupe schématique du système d'enclumes toroïdales : le symbole (\$) désigne l'échantillon et (g) le joint ("gasket") assurant le confinement du milieu transmetteur. (D'après les références [22] (a),(c) et le "ILL Yellowbook" (b).)

d'investigation et rendre possibles des études neutroniques complémentaires de celles réalisées sur les sources de rayonnement synchrotron. Ces techniques font l'objet des deux prochaines sections.

\subsection{Les presses "Paris-Édimbourg"}

Convaincue que la clé du développement des mesures de neutrons à haute pression résidait dans une augmentation des volumes d'échantillon, l'équipe de Besson va choisir d'utiliser la technique des "enclumes toroïdales" expérimentée une dizaine d'années plus tôt à l'Institut des Hautes Pressions de Troitsk [20] pour concevoir le dispositif popularisé depuis sous le nom de "Cellule/Presse ParisÉdimbourg" [7]. Adopté aujourd'hui par de nombreux centres de neutrons, celui-ci est couramment utilisé pour les mesures de diffraction, et un appareil en temps de vol (PEARL) lui est même spécialement consacré sur la source ISIS du Rutherford Appleton Laboratory. La pression maximale atteinte ainsi que le volume d'échantillon dépendent du type d'enclume utilisé. Les mesures à $26 \mathrm{GPa}$ sur la phase VII de la glace deutériée (groupe d'espace $P n \overline{3} m$ ) présentées sur la Figure 15 ont été effectuées à la 


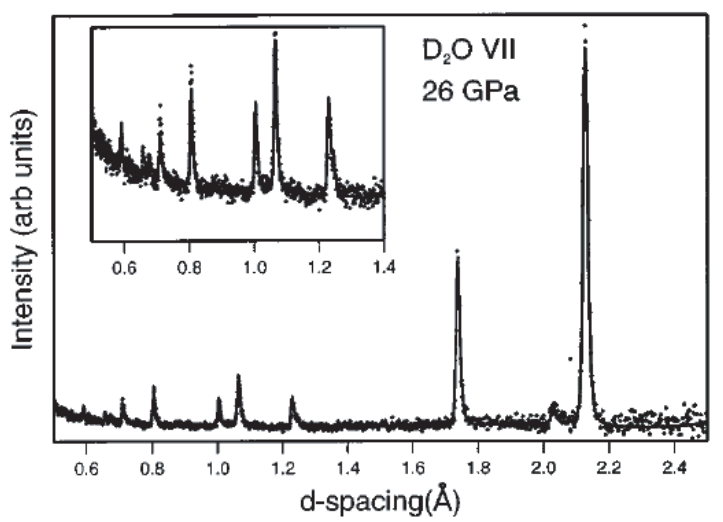

Figure 15. Diffractogramme de la phase VII de $\mathrm{D}_{2} \mathrm{O}$ sous $26 \mathrm{GPa} a ̀ t=300 \mathrm{~K}$ mesuré en temps de vol sur l'appareil PoLARIS à ISIS (d'après le référence [22]).
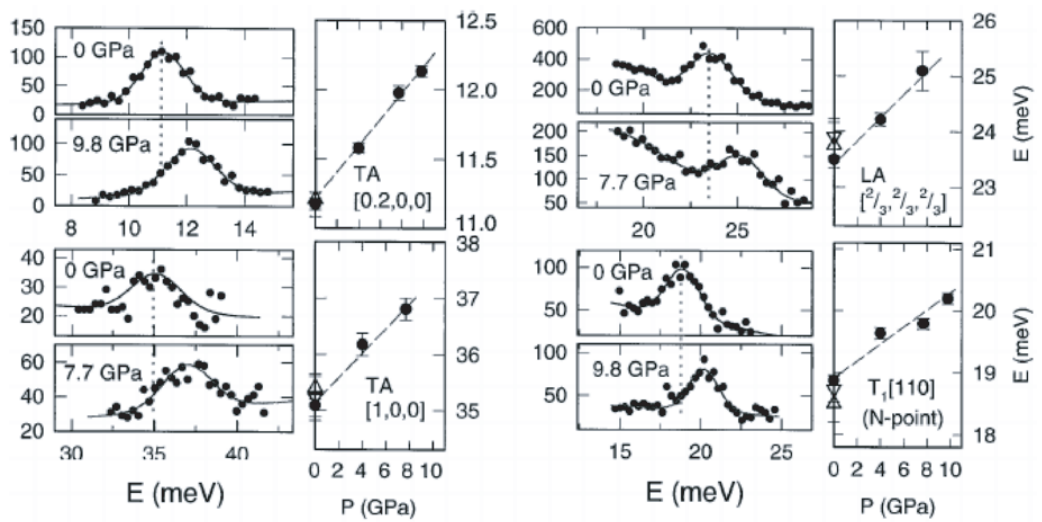

Figure 16. Dépendance en pression de quatre modes de phonons dans le fer cubique centré (d'après la référence [23]).

température ambiante dans des enclumes en diamant polycristallin fritté avec un échantillon de $35 \mathrm{~mm}^{3}$ [22]. Des progrés ultérieurs dans le mode d'encapsulation de l'échantillon ont permis de travailler en milieu liquide méthanol-éthanol, donc avec une meilleure hydrostaticité, jusqu'à $9 \mathrm{GPa}$ [26].

Une série d'expériences réalisées sur un spectromètre trois-axes thermique au LLB (Saclay) ont démontré que des mesures de diffusion inélastiques sur monocristal sont également possibles jusqu'à des pressions d'au moins $10 \mathrm{GPa}$, pourvu que le phénomène étudié posséde une section efficace suffisante. L'exemple montré ici [22] est celui du fer, qui présente une transition structurale martensitique sous $P_{c}=11 \mathrm{GPa}$ entre une phase basse pression cubique centrée $(\alpha)$ et une phase haute pression hexagonale compacte $(\epsilon)$. Alors qu'on s'attendait, dans le modéle de Burgers, à l'existence d'effets prétransitionnels sur la dynamique de réseau, les données (Fig. 16) montrent au contraire une augmentation monotone des fréquences des différents modes de phonons jusqu'à une pression de $10 \mathrm{GPa}$ voisine de $P_{c}$. Ce résultat en apparence paradoxal va dans le sens de prédictions théoriques attribuant un rôle dominant au magnétisme du fer dans le mécanisme de la transition.

\subsection{Les cellules "Kourtchatov-LLB"}

Forts de leur expérience sur les cellules à enclumes en diamant, les chercheurs de l'Institut Kourtchatov vont eux aussi s'efforcer d'accroître la taille de leurs échantillons, mais sans renoncer aux avantages 


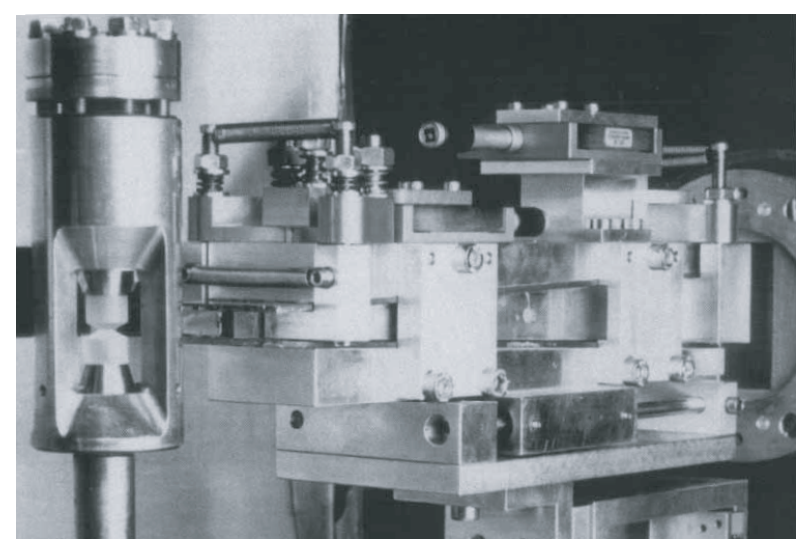

Figure 17. Chambre "Kourtchatov-LLB" avec ses enclumes en saphir sur le diffractométre G6-1 MICRO du LLB muni du système à miroirs focalisants Focus 1 (d'après la référence [15]).

de ce type d'appareil en matière d'encombrement, de force appliquée et de bruit de fond. La clé pour y parvenir a été la mise au point d'enclumes en saphir monocristallin dans lesquelles le volume d'échantillon peut aller jusqu'au millimétre cube. D'abord utilisées pour des études structurales sur des hydrures jusqu'à $13 \mathrm{GPa}$ [13], ces cellules ont ensuite été perfectionnées par Goncharenko [15] dans le cadre d'une collaboration avec le LLB à Saclay, et déclinées en différentes versions ("cellules Kourtchatov-LLB") à la fois pour des mesures sur poudres et sur monocristaux. Du fait de leur compacité, elles se prêtent particulièrement bien aux mesures à très basse température et sous fort champ magnétique. Dotées de larges ouvertures, elles ne produisent pratiquement aucune diffusion parasite et la seule source résiduelle est le joint d'étanchéité ("gasket") dont le matériau (aluminium, cuivre-béryllium, cuivre-nickel) est choisi en fonction des conditions souhaitées. On dispose en outre, grâce à la transparence des enclumes, d'une mesure précise de la pression déduite du déplacement d'une raie de fluorescence du rubis dont un grain est placé au voisinage immédiat de l'échantillon. Dans le cas des monocristaux, l'utilisation d'un milieu transmetteur liquide (méthanol-éthanol) permet de préserver l'intégrité de l'échantillon pendant la montée en pression.

En dépit de tous ces avantages, l'utilisation de cellules avec des échantillons de taille aussi réduite impose de sérieuses contraintes au niveau de la mesure neutronique. Ceci a conduit à spécialiser le diffractométre de poudre G6-1 du LLB pour les mesures sous très haute pression, en l'équipant notamment du système de focalisation variable à supermiroirs en Ni-Ti (Focus) représenté sur la Figure 17. La technique originale des enclumes diamant a aussi bénéficié de ces améliorations et des pressions record proches de $50 \mathrm{GPa}$ ont pu être atteintes dans des mesures sur le composé GdAs [14].

La Figure 18 présente une étude [29] de l'ordre magnétique dans le composé USb, de structure cubique $\mathrm{NaCl}$, oú l'hybridation entre les états électroniques $5 f$ et la bande de conduction est connue pour jouer un rôle essentiel. Des études antérieures avaient établi que la structure à pression nulle correspond à une forme particulière d'antiferromagnétisme dans laquelle les moments des atomes d'uranium sont non-colinéaires, pointant suivant les différentes directions $\langle 111\rangle$ du cube. Celle-ci est qualifiée de "triple- $k$ " parce que sa description fait intervenir simultanément, pour un même domaine magnétique, trois composantes de Fourier associées aux trois vecteurs de propagations $\boldsymbol{k}_{x}=(1,0,0)$, $\boldsymbol{k}_{y}=(0,1,0)$ et $\boldsymbol{k}_{z}=(0,0,1)$. Les résultats obtenus sur un monocristal à une pression de $3,8 \mathrm{GPa}$ (Fig. 18(a)) montrent l'existence d'une seconde transition au-dessous de $T_{N}$ dans laquelle seules les intensités relatives des pics de Bragg associés à $\boldsymbol{k}_{x}, \boldsymbol{k}_{y}$ et $\boldsymbol{k}_{z}$ sont modifiées. Cet effet a été attribué à un changement de structure de triple- $k$ (un seul domaine avec trois composantes de Fourier) à simple- $k$ (trois domaines avec chacun une seule composante de Fourier). La population inégale de ces domaines, due à l'existence de faibles contraintes uniaxiales à l'intérieur de la cellule, conduit à des 

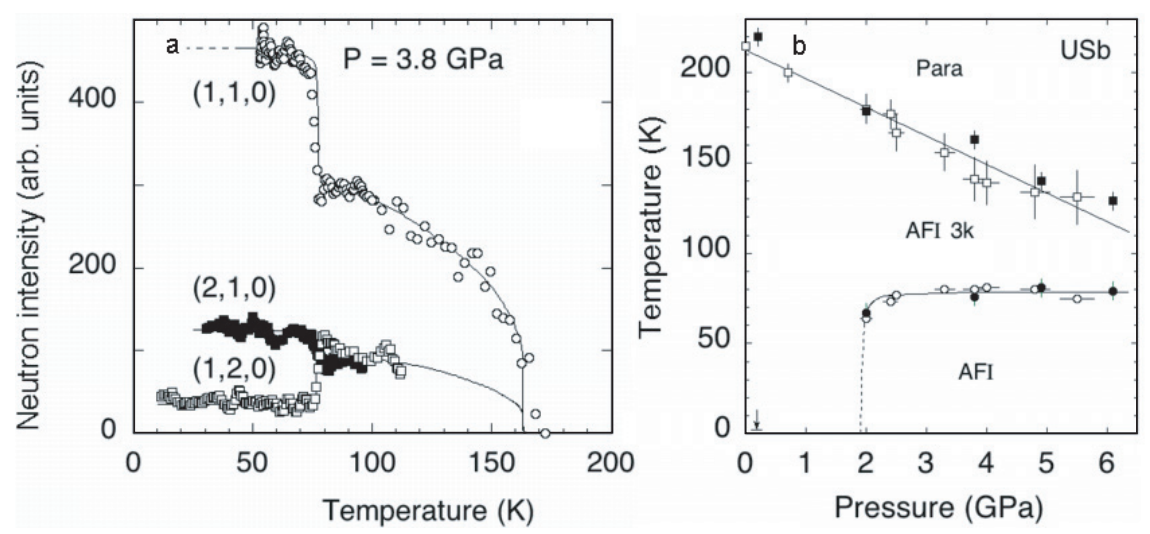

Figure 18. Étude de l'ordre magnétique dans le composé USb sous pression, réalisée dans une cellule "Kourtchatov-LLB" à enclumes en saphirs : (a) variation d'intensité de trois pics de Bragg antiferromagnétiques en fonction de la température ; (b) diagramme de phase magnétique sous pression. (D’après la référence [29].)
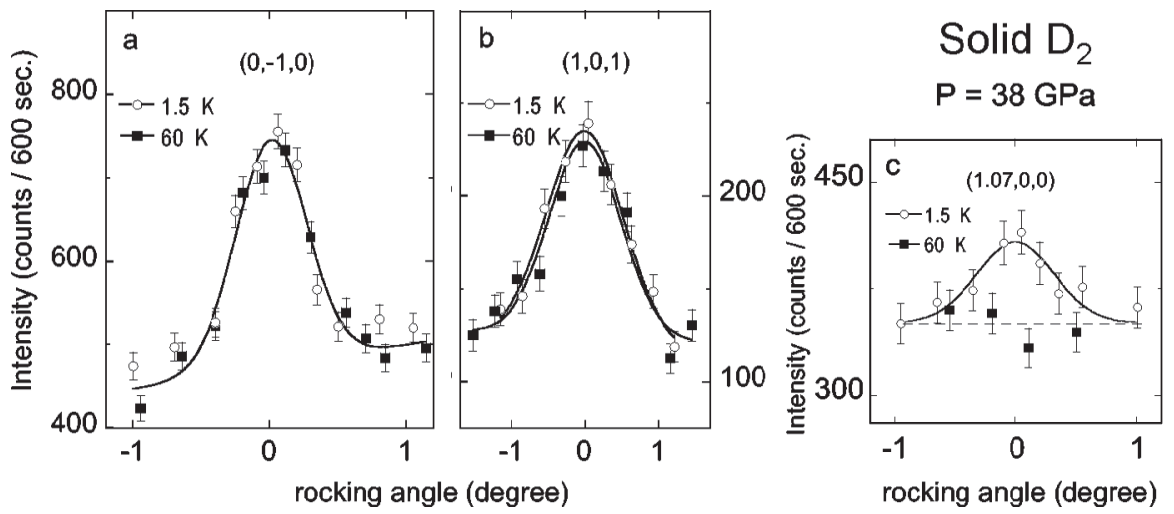

Figure 19. Phase de symétrie brisée dans le deutérium solide étudiée par diffraction de neutrons dans une cellule "Kourtchatov-LLB" sous pression hydrostatique (milieu transmetteur d'hélium liquide) de $38 \mathrm{GPa}$ : (a), (b) pics de Bragg de la structure hexagonale mesurés au-dessus et au-dessous de la température de transition; (c) pic de surstructure incommensurable. (D’après la référence [16].)

rapports d'intensités incompatibles avec une structure monodomaine préservant la symétrie cubique. Le diagramme de phase tracé sur la Figure 18(b) pour des pressions allant jusqu'à 6 GPa est en excellent accord avec les résultats déduits des mesures de résistivité [8].

Dans une gamme de pressions beaucoup plus élevées, on citera les récentes études sur les transitions structurales dans les solides quantiques moléculaires, réalisées grâce à une nouvelle génération de cellules "hybrides" à enclumes en diamant [17]. Dans le cas du deutérium solide, la combinaison d'expériences de diffraction de rayons X sur synchrotron (ESRF Grenoble) et de neutrons au LLB Saclay (Fig. 19) a permis de démontrer que, contrairement à certaines prédictions théoriques, la "phase de symétrie brisée" qui existe au-dessus de $28 \mathrm{GPa}$ ne présente pas de composante orthorhombique, mais correspond à un ordre local orientationnel des molécules $\mathrm{D}_{2}$ sur le réseau original hexagonal compact [16]. L'existence dans cette structure de phénomènes de frustration induit en outre une modulation incommensurable, clairement indiquée par un pic de surstructure, comme on le voit sur la Figure 19 pour une pression appliquée de $38 \mathrm{GPa}$. 


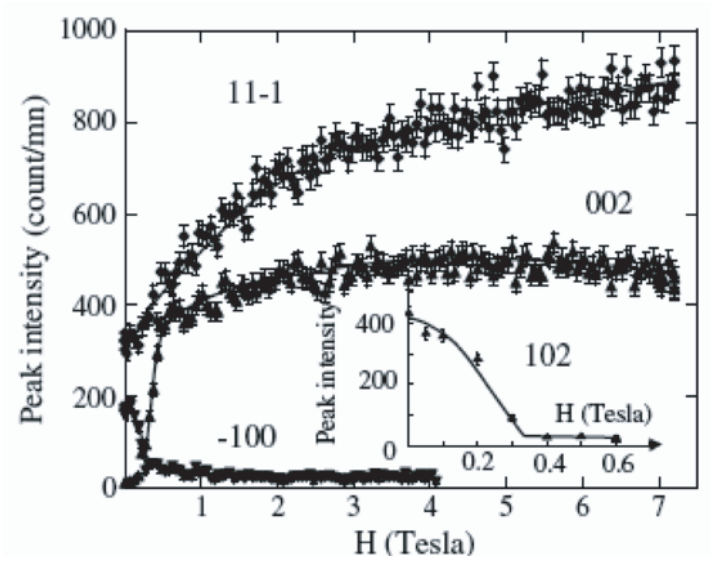

Figure 20. Effet d'un champ magnétique à très basse température $(T=140 \mathrm{mK})$ sur la phase $\mathrm{AF}$ du composé à structure pyrochlore $\mathrm{Tb}_{2} \mathrm{Ti}_{2} \mathrm{O}_{7}$ obtenue par l'application simultanée d'une pression hydrostatique et d'une contrainte uniaxiale (d'après la référence [30]).

\section{CONCLUSION}

Au vu de ce rapide survol, il ne fait pas de doute que le domaine des conditions extrêmes est encore promis à d'importants développements dans les prochaines années. Tandis que des techniques autrefois réservées à de rares experts font aujourd'hui partie de l'infrastructure expérimentale offertes aux utilisateurs des grands centres de neutrons, des projets ambitieux voient le jour pour tenter d'atteindre de nouvelles limites, que ce soit dans la production d'ultra-basses températures, de très forts champs magnétiques ou de pressions extrêmes, souvent à travers des synergies entre sources de neutrons et de rayons $\mathrm{X}$. Le pouvoir pénétrant des neutrons dans la matière reste à cet égard un avantage majeur, même si le champ d'étude couvert par les sources de rayonnement synchrotron ne cesse de s'étendre.

Dans de nombreux cas, les problèmes abordés nécessitent de combiner les effets de plusieurs paramétres thermodynamiques. Cette tendance est bien illustrée par l'étude du liquide de spin à structure pyrochlore $\mathrm{Tb}_{2} \mathrm{Ti}_{2} \mathrm{O}_{7}$ [30] dans lequel il faut, pour surmonter les effets de frustration et induire un ordre $\mathrm{AF}$ à longue distance, appliquer simultanément une pression hydrostatique de 2,8 GPa (augmentation du superéchange) et une contrainte uniaxiale de $0,3 \mathrm{GPa}$ (abaissement de la symétrie locale). La Figure 20 montre que cet ordre est rapidement supprimé, à une température de $140 \mathrm{mK}$, par l'application d'un champ magnétique, laissant place à un "canting" progressif des moments qui se poursuit jusqu'au champ maximal atteint de $7 \mathrm{~T}$.

Pour finir, il est intéressant de noter que les réalisations les plus remarquables parmi celles qui viennent d'être décrites résultent de la conjonction d'une forte motivation au sein d'une communauté scientifique et d'un apport de compétence, de créativité — et de ténacité ! — de la part de spécialistes maîtrisant les techniques appropriées. Il est donc essentiel de maintenir les unes comme les autres au plus haut niveau pour garantir les développements futurs.

\section{Remerciements}

Dans le préparation de ce texte, j'ai bénéficié de discussions avec M. Meißner et P. Schmeibidl sur les installations de forts champs magnétiques existantes ou en projet au HMI Berlin. Je remercie également I. N. Goncharenko, S. Klotz, M. Kohgi, E. Leliévre-Berna, I. Mirebeau et J. Bossy pour les informations qu'ils m'ont fournies et pour leurs commentaires sur le présent manuscrit. Différentes illustrations sont 
reproduites ici avec l'aimable permission de l'Institut Laue-Langevin, du Hahn-Meitner Institut, de l'Institut Paul Scherrer, et des sociétés AS Scientific Products, l'Air Liquide et Janis.

\section{Références}

[1] Badurek, G., Rader, S. and Grossinger, R., J. Magn. Magn. Mater. 140-144 1533 (1995).

[2] Bailey, I. F., Z. Kristallogr. 218, 84 (2003).

[3] Benoît, A., Bossy, J., Flouquet, J. and Schweizer, J., J. Phys. Lett. (Paris) 46, 923 (1985).

[4] Benoît, A. and Pujol, S., Physica B 169, 457 (1991).

[5] Benoît, A., Caussignac, M. and Pujol, S., Physica B 197, 48 (1994).

[6] Neutron scattering instrumentation at the research reactor BER II., Berlin Neutron Scattering Center - BENSC (2007).

[7] Besson, J. M., Nelmes, R. J., Hamel, G., Loveday, J. S., Weill, G. and Hull, S., Physica B: Condensed Matter 180-181, 907 (1992).

[8] Braithwaite, D., Goncharenko, I. N., Mignot, J.-M., Ochiai, A. and Vogt, O., Europhys. Lett. 35, 121 (1996).

[9] Brochier, D., Institut Laue-Langevin, Grenoble, 74/77 (1977).

[10] Dubinin, V. P., Voinov, A. M., Voinov, M. A. and Kolesov V. F., Proceedings of the 27th International Meeting on Reduced Enrichment for Research and Test Reactors (Boston, USA, 2005) URL: http://www.rertr.anl.gov/RERTR27/PDF/S12-9_Dubinin.pdf.

[11] Enderle, M., Rønnow, H. M., McMorrow, D. F., Regnault, L.-P., Dhalenne, G., Revcholevschi, A., Vorderwisch, P., Schneider, H., Smeibidl, P. and Meißner, M., Phys. Rev. Lett. 87, 177203 (2001).

[12] Georgiev, D., Nietz, V. V., Petukhova, T. B., Sirotin, A. P., Varenik, G. A. and Yakovlev, A. A., J. Neutron Res. 5, 109 (1997).

[13] Goncharenko, I. N., Glazkov, V. P., Irodova, A. V., Lavrova, O. A. and Somenkov, V. A., J. Alloys Comp. 179, 253 (1992).

[14] Goncharenko, I. N., Mirebeau, I. and Ochiai, A., Hyperfine Interactions 128, 225 (2000).

[15] Goncharenko, I. N., High Press. Research 24, 193 (2004).

[16] Goncharenko, I. and Loubeyre, P., Nature 435, 1206 (2005).

[17] Goncharenko, I. N., High Press. Research 27, 183 (2007).

[18] Jyrkkiö, T. A., Huiku, M. T., Siemensmeyer, K. and Clausen, K. N., J. Low Temp. Phys. 74, 435 (1989).

[19] Kaneko, K., Metoki, N., Matsuda, T. D., Kuwahara, K., Kohgi, M., Shiina, R., Mignot, J.-M., Gukasov, A. and Bernhoeft, N., Physica B 378-380, 189 (2006).

[20] Khvostantsev, L. G., High Temp., High Press. 16, 165 (1984).

[21] $\mathrm{Ni}$, en poussant le concept (justement) à l'extrême, "l'ultramicroscopique, le très vaste, l'extrêmement bref, le très lointain, l'incroyablement rare, le très chaud, l'ultradiscret", comme a pu le suggérer E. Klein [Les mesures de l'extrême, site Internet CEA, octobre 2006 (non publié)]

[22] Klotz, S., Besson, J. M., Hamel, G., Nelmes, R. J., Loveday, J. S., Marshall, W. G. and Wilson, R. M., Appl. Phys. Lett. 66, 1735 (1995).

[23] Klotz, S. and Braden, M., Phys. Rev. Lett. 85, 3209 (2000).

[24] Knorr, K., Futterer, K., Annighofer, B. and Depmeier, W., Rev. Sci. Instrum. 68, 3817 (1997).

[25] Kuhs, W. F., Hensel, E. and Bartels, H., J. Phys.: Condens. Matt. 17, S3009 (2005).

[26] Marshall, W. G. and Francis, D. J., J. Appl. Crystall. 35, 122 (2002).

[27] McWhan, D. B., Vettier, C., Youngblood, R. and Shirane, G., Phys. Rev. B 20, 4612 (1979).

[28] Meissner, Michael and Smeibidl, Peter, Neutron News 12, 12 (2001).

[29] Mignot, J.-M. and Goncharenko, I. N. and Braithwaite, D. and Vogt, O., J. Phys. Soc. Jpn. Suppl. $B$ 65, 91 (1996).

[30] Mirebeau, I. and Goncharenko, I. N., J. Phys.: Condens. Matter 17, S771 (2005). 
[31] Nietz, V., J. Magn. Magn. Mater. 260, 84 (2003).

[32] Nojiri, H., Uchi, M., Watamura, S., Motokawa, M., Kawai, H., Endoh, Y. and Shigeoka, T., J. Phys. Soc. Jpn. 60, 2380 (1991).

[33] Nojiri, H., (communication personnelle)

[34] Pobell, F., Matter and Methods at Low Temperatures (Springer, Berlin, 1992).

[35] Sears, V. F., Neutron News 3, 26 (1992).

[36] Sidorov, V. A. and Sadykov, R. A., J. Phys.: Condens. Matter 17, S3005 (2005).

[37] Somenkov, V. A., J. Phys.: Condens. Matter 17, S2991 (2005).

[38] Suzuki, H., Ohtsuka, T., Kawarazaki, S., Kunitomi, N., Moon, R. M. and Nicklow, R. M., Solid State Commun. 49, 1157 (1984).

[39] Tayama, T., Sakakibara, T., Sugawara, H., Aoki, Y. and Sato, H., J. Phys. Soc. Jpn. 72, 1516 (2003).

[40] Tuoriniemi, J. T., Nummila, K. K., Vuorinen, R. T., Lounasmaa, O. V., Metz, A., Siemensmeyer, K., Steiner, M., Lefmann, K., Clausen, K. N. and Rasmussen, F. B., Phys. Rev. Lett. 75, 3744 (1995).

[41] Varga, T., Wilkinson, A. P. and Angel, R. J., Rev. Sci. Instrum. 74, 4564 (2003).

[42] $\mathrm{Ni}$, pour pousser le concept (justement) à l'extrême, "l'ultramicroscopique, le très vaste, l'extrêmement bref, le très lointain, l'incroyablement rare, le très chaud, l'ultradiscret", comme le suggère E. Klein [Les mesures de l'extrême, site Internet CEA, octobre 2006 (non publié)] 